\title{
Resolving the Diagnostic Dilemma of Clear Cell Odontogenic Carcinoma Using Special Stains: A Case Report
}

Lavanya Mallika ${ }^{1}$, SV Sowmya ${ }^{2}$, Rajanikanth B Rajaram ${ }^{3}$, Anand Devaraj $^{4}$, Roopa S Rao ${ }^{5}$, Kavitha Prasad ${ }^{6}$, Dominic Augustine ${ }^{7}$, Vanishri C Haragannavar ${ }^{8}$

\begin{abstract}
Aim: To resolve the diagnostic dilemma of clear cell odontogenic carcinoma (CCOCa) using various special stains.

Background: Clear cell odontogenic carcinoma is a rare malignant odontogenic tumor with a female predilection, occurring predominantly in the mandible. It is an aggressive tumor with frequent recurrences (55\%) and high metastatic potential (31\%).

Case description: Herein, we report a case of an unusual extensive mandibular tumor mass in 49-year-old male patient who posed diagnostic challenges due to similar microscopic features of oral squamous cell carcinoma (OSCC), clear cell mucoepidermoid carcinoma (CCMEC), hyalinizing clear cell carcinoma (CCC), epithelial myoepithelial carcinoma (EMC), clear cell variant of calcifying epithelial odontogenic tumor (CCCEOT), and clear cell odontogenic ghost cell tumor (CCOGCT).

Conclusion: The use of appropriate special stains like mucicarmine, Van Gieson, and Periodic Acid Schiff with and without diastase paved the way toward confirmatory diagnosis.

Clinical significance: Good knowledge and right practice of various special staining techniques in histopathology provides a rapid and reproducible result with better diagnosis.

Keywords: Clear cell odontogenic carcinoma, Mandibular neoplasm, Special stains.

World Journal of Dentistry (2019): 10.5005/jp-journals-10015-1648
\end{abstract}

\section{INTRODUCTION}

Clear cell odontogenic carcinoma (CCOCa) is a rare intraosseous odontogenic neoplasm of the jaws. It was first defined as clear cell odontogenic tumor or clear cell ameloblastoma by Hansen and Waldron et al. in 1985 considering its locally destructive nature. ${ }^{1}$ In 1992, as the malignant potential was not yet determined, WHO described this entity as a benign tumor. However in 2003, Reichart and Philipsen suggested the term CCOCa. This was supported by WHO in 2005 due to its infiltrative, recurring, regional, and distant metastatic potential. ${ }^{1,2}$ However, biological and prognostic behavior of CCOCa remains illusory because of dearth of cases and hence the treatment planning remains uncertain. In this report, we discuss the clinical, histopathological, and diagnostic features of a rare case of CCOCa in a 49-year-old male patient presenting with a large swelling in the mandible, crossing the midline. ${ }^{1-3}$

\section{Case Description}

A middle-aged male patient of 49 years reported to the outpatient department of Faculty of Dental Sciences (FDS), MS Ramaiah University of Applied Sciences, with a chief complaint of painful swelling in the lower jaw from the past 2 months. Patient was apparently well 2 months ago, when he developed a slow-growing swelling associated with dull aching continuous pain on the left side of his lower jaw. He consulted a local dentist where he was advised panoramic radiograph and underwent extraction of left mandibular second premolar under local anesthesia for the same complaint. There was no reduction in swelling following extraction and patient was referred to FDS for further investigation and needful.

Extraoral examination revealed a diffuse swelling over the lower jaw extending from left side of the cheek, crossing the midline up to the right corner of the mouth in the transverse direction, involving
1,2,5,7,8 Department of Oral Pathology and Microbiology, MS Ramaiah University of Applied Sciences, Bengaluru, Karnataka, India

${ }^{3,4,6}$ Department of Oral and Maxillofacial Surgery, MS Ramaiah University of Applied Sciences, Bengaluru, Karnataka, India

Corresponding Author: Lavanya Mallika, Department of Oral Pathology and Microbiology, MS Ramaiah University of Applied Sciences, Bengaluru, Karnataka, India, Phone: +91 9095231745 , e-mail: drlavsmdsop92@gmail.com

How to cite this article: Mallika L, Sowmya SV, Rajaram RB, et al. Resolving the Diagnostic Dilemma of Clear Cell Odontogenic Carcinoma Using Special Stains: A Case Report. World J Dent 2019;10(4):316-320.

Source of support: Nil

Conflict of interest: None

the lower third of the face. The swelling was approximately $8 \times 3 \mathrm{~cm}$ in size. On palpation, the swelling was firm, nontender, and non-fluctuant with no discharge. The overlying skin appeared normal. The submental and submandibular lymph nodes were palpable and tender, firm, and fixed to underlying structures.

Intraoral examination exhibited a well-defined swelling involving the mandible measuring approximately $7 \times 3 \mathrm{~cm}$. The swelling extended from the mesial aspect of 37-46 and appeared to involve the height of mandible. The swelling appeared lobulated expanding buccal and lingual cortices and extending into the floor of the mouth (Fig. 1A). An exophytic soft sessile erythematous growth with ulceration was observed in relation to 35 region.

\section{Investigations}

Panoramic radiograph revealed a multiloculated mass showing irregular margins with loss of normal bone architecture extending 

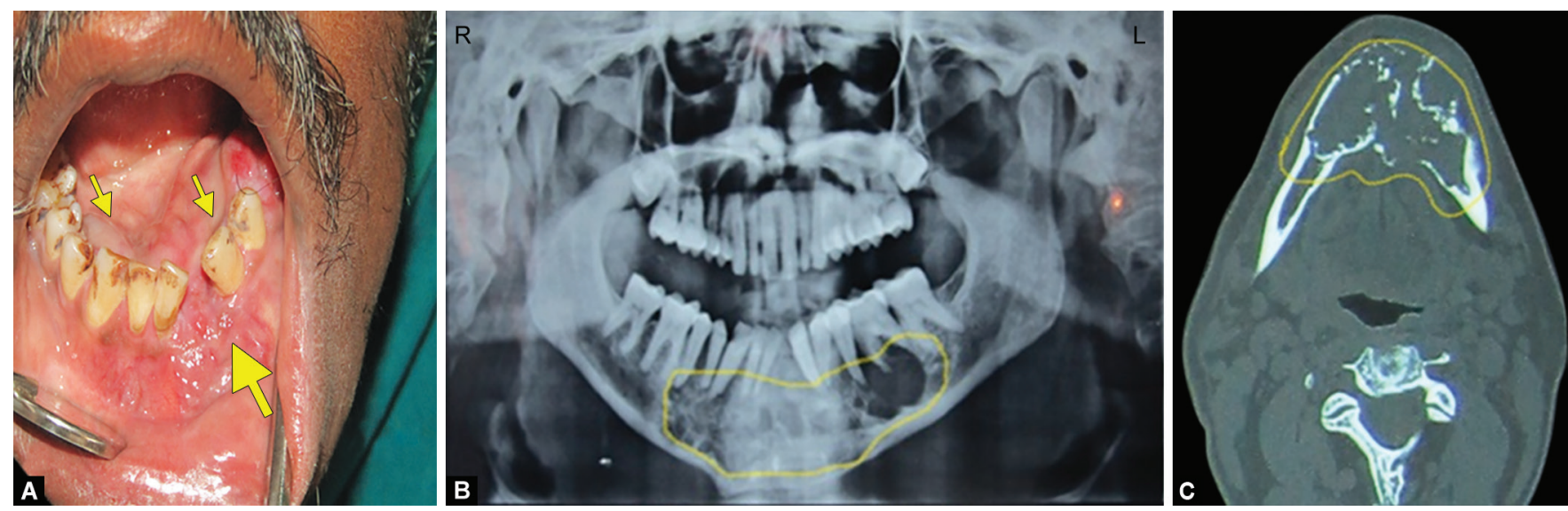

Figs 1 A to C: (A) Intraoral photograph showing an exophytic erythematous growth with ulceration in relation to 35 ; (B) Panoramic radiograph showing a multiloculated mass with irregular margins and loss of normal bone architecture extending from 37 to 45 regions; (C) CT scan showing buccal and lingual cortical expansion with erosion of normal bone from 37 to 45 regions

from 37 to 46 region (Fig. 1B). Computed tomography (CT) scan showed a multilocular radiolucent lesion with irregular margins. Buccal and lingual cortical expansion with erosion of normal bone was also observed (Fig. 1C).

Routine hematological investigations were performed and found to be within physiological limits. An incisional biopsy was performed from the anterior mandible in relation to 31, 32, 41 and 42 regions under local anesthesia. The excised gross specimen was brownish gray in color, irregular in shape, firm in consistency, and measured approximately $3 \times 0.4 \times 0.5 \mathrm{~cm}$ (Fig. 2).

\section{Histopathological Findings}

On microscopic examination, odontogenic epithelium was seen proliferating in the form of sheets and islands with interconnecting strands and cords in a fibrous, hyalinized stroma (Fig. 3A). Few odontogenic follicles comprising of peripheral tall columnar ameloblast-like cells showing hyperchromatic nuclei with reversal of polarity were observed (Fig. 3B). The center of the follicles showed biphasic population of round, polyhedral pleomorphic squamous cells and clear cells with small dark peripherally placed nuclei (Fig. 3C). Squamous metaplasia, keratin pearls (Figs 3D and E), and areas of cystic degeneration were also observed. Majority of the sections showed hypercellularity with round to polygonal

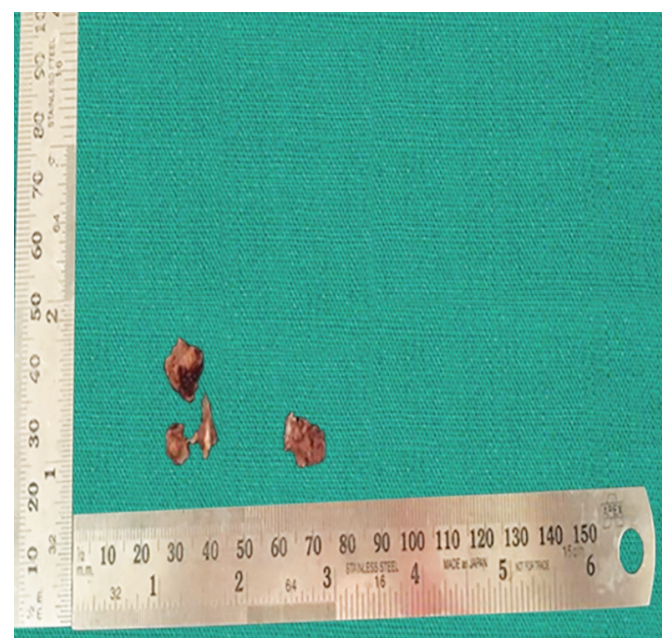

Fig. 2: Gross specimens of CCOCa cells having eosinophilic cytoplasm, hyperchromatic nuclei, and pleomorphism arranged in sheets and islands separated by dense fibrous septae. Eosinophilic sheets of dentin-like material were present within the tumor cells and fibrous stroma (Fig. 3F). The stroma was extensively hyalinized with foci of mature bone and moderate vascularity. With the above histopathological features, a diagnosis of a clear cell lesion was inferred. The diagnoses of salivary gland malignancy or odontogenic neoplasms were considered with the observed features. Hence, special stains like, mucicarmine, Van Gieson, and Periodic acid Schiff (PAS) staining with and without diastase, were performed to identify mucin and glycogen, respectively. The present case was negative for mucicarmine (Fig. 4A) and Van Gieson (Fig. 4B) and positive for PAS with (light pink) and without (magenta) diastase (Figs $4 C$ and D). With the histopathological features observed and special stains performed, the diagnosis of CCOCa was confirmed and surgical resection was planned.

\section{Discussion}

Clear cells are either epithelial or mesenchymal cells associated with physiological and pathological conditions. These cells display pale or clear cytoplasm with a definite nucleus. The clear cytoplasm results from fixation artifact changes, paucity of cellular organelles, and cytoplasmic accumulation of various substances such as water, glycogen, mucin or lipid, and intermediate filaments. Any benign or malignant tumor of epithelial, mesenchymal, and hematopoietic origin can show clear cells but has been very rarely reported in the head and neck regions. When the clear cells predominate, ambiguity prevails in obtaining an accurate diagnosis of these lesions. ${ }^{2,4}$ There are numerous histochemical and molecular techniques available to arrive at quick and accurate results. Among them, the histostaining technique with special stains is inexpensive and gives rapid and reproducible results with better diagnosis. However, various special staining techniques are available to detect the intracellular secretory components like glycogen, lipid, or mucin content in clear cells. The special stains are PAS with or without diastase, mucicarmine, Sudan black, van Gieson, crystal violet, Alcian blue, etc. ${ }^{5-7}$

The odontogenic tumors originate from remnants of dental lamina and cell rests of Malassez, which are physiological clear cells. The cytoplasm of these cells is rich in glycogen that is a 

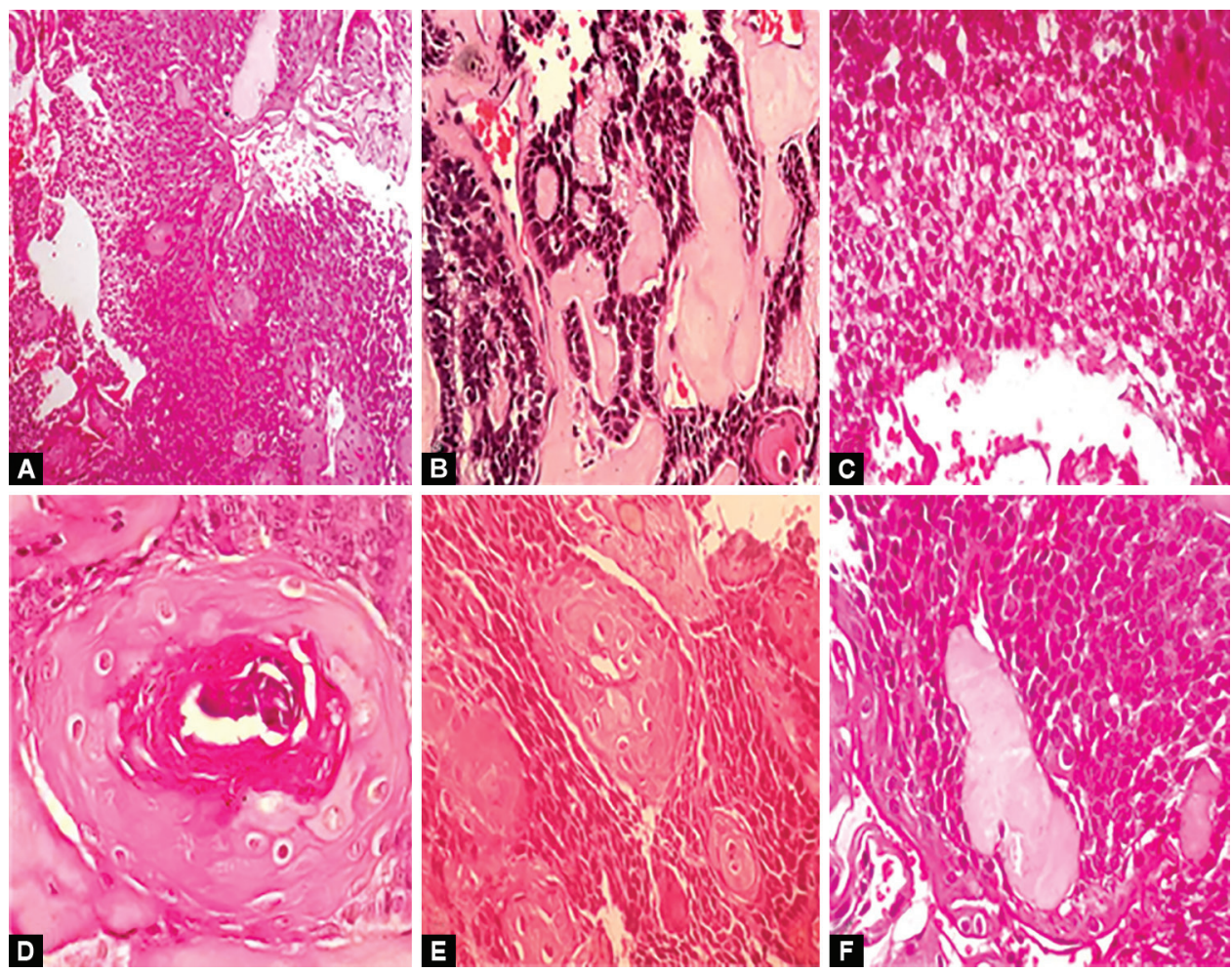

Figs $3 \mathrm{~A}$ to F: Photomicrographs of H\&E stained sections of CCOCa; (A) Odontogenic epithelium proliferating in the form of sheets $(\times 100)$; $(B)$ Odontogenic follicles comprising of peripheral tall columnar ameloblast-like cells $(\times 400)$; (C) Clear cells with dark peripherally placed nuclei; (D) Keratin pearl formation (X400); (E) Squamous metaplasia (X400); (F) Dentin-like material $(\times 400)$

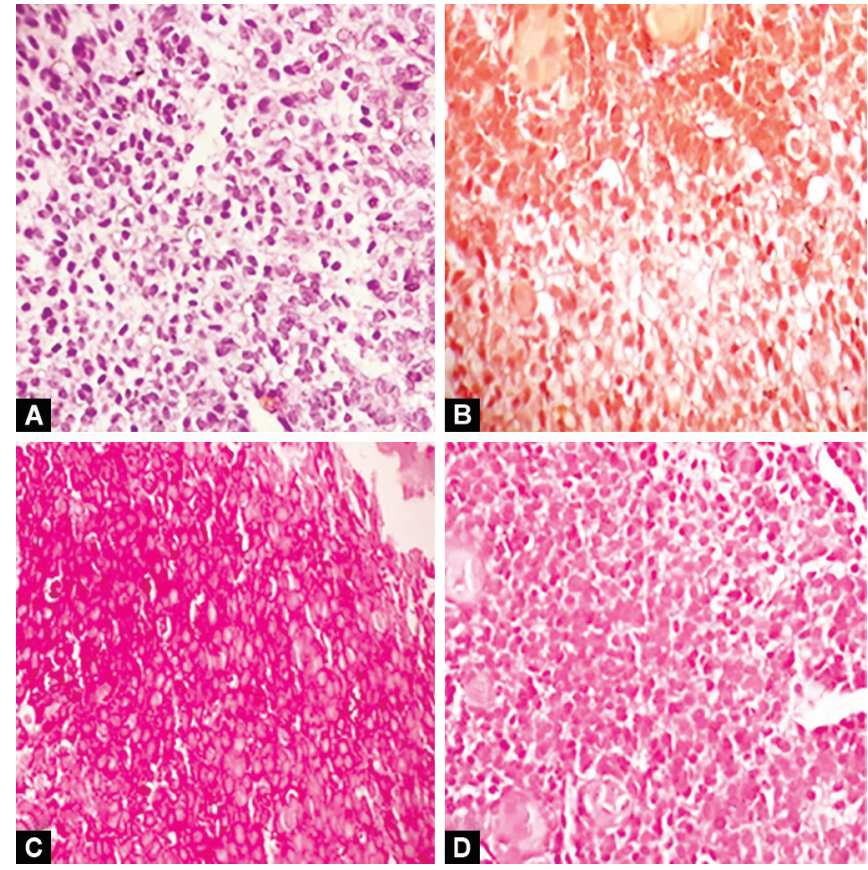

Figs 4A to D: Photomicrographs of CCOCa showing clear cells that are negative for (A) mucicarmine and (B) Van Gieson staining ( $\times 400)$; positive for (C) PAS without diastase (magenta color) $(\times 400)$; and (D) PAS with diastase staining (light pink) $(\times 400)$

mucopolysaccharide and positive for PAS. The presence of glycogen is also determined by digesting the glycogen using the diastase enzyme, represented as diastase sensitivity.
Clear cell odontogenic carcinomas that have been reported so far are in female patients; ${ }^{3,5,8,9}$ however, in the present case, the patient is male. The case presented in this report was characterized by an intrabony swelling with exophytic growth extending to the contralateral side with wide involvement from premolar on one side (45) to molar on other side (37). Such extensive lesions have been minimally reported in the literature till date. ${ }^{3,9,10}$ The histopathological features in the present case exhibited keratin pearl formation and dentin-like material, which is a rare presentation observed in CCOCas. The keratin formation in the present case was due to the squamous metaplasia and dentin-like material suggestive of epithelial-mesenchymal induction. ${ }^{11}$

Based on the clinical and radiographic findings, ameloblastoma, odontogenic keratocyst, and metastatic tumors from kidney, thyroid, and prostate may be considered in the differential diagnoses. Histopathological findings of the incisional biopsy showed biphasic squamous and clear tumor cells. However, clear cell neoplasms are exceptionally uncommon in the head and neck region. The present histopathological features prompted the inclusion of clear cell mucoepidermoid carcinoma (CCMEC), hyalinizing clear cell carcinoma (CCC), epithelial myoepithelial carcinoma (EMC), clear cell variant of calcifying epithelial odontogenic tumor (CCCEOT), CCOCa, and clear cell odontogenic ghost cell tumor (CCOGCT) as the differential diagnosis. ${ }^{4}$

Oral squamous cell carcinoma (OSSC) is the most common malignant epithelial tumor of all oral neoplasms. It occurs frequently on tongue, lips, and floor of the mouth. Histopathologically, it shows various dysplastic features like hyperchromatic nuclei, nuclear and cytoplasmic pleomorphism, increased nuclear cytoplasmic ratio, keratin pearl formation, loss of polarity, and mitotic figures with varying degree in differentiation. However, the present case 
showed keratin pearl formation and squamous metaplasia with odontogenic islands. Keratin pearl formed in odontogenic tumors is not surrounded by dysplastic cells and termed as "benign keratin pearl." However, in OSCC, keratin is laid down in concentric pattern or whorl-shaped accumulations by malignant squamous cells, which is represented as "malignant keratin pearl." In the present case, keratin pearl formed is in the benign form, which helped in excluding OSCC.

Clear cell mucoepidermoid carcinoma is a very rare lesion and occurs more commonly in the mandible than the maxilla. Clear cells may lead to diagnostic dilemma in MEC. Microscopically, it shows infiltrative, multiple cystic spaces lined by mucous, epidermoid, intermediate, and clear cells. The clear cells consist of glycogen, exhibit pale basophilic foamy cytoplasm, and are positive for PAS staining with diastase sensitivity (light pink). Mucin in cystic spaces shows positivity for Alcian blue and mucicarmine staining. $8,12,13$ Although the present case was positive for PAS and diastase labile, the diagnosis of CCMEC was excluded due to negative mucicarmine staining (Fig. 4A).

Hyalinizing CCC is a rare minor salivary gland tumor of the oral cavity frequently occurring in the tongue with a female predilection, in the fifth to seventh decades of life. It is comprised of glycogen-rich clear cells in the form of trabeculae, cords, islands, and nests with abundant hyalinized stroma. The rich glycogen content of the clear cells is demonstrated by PAS and not with mucin. ${ }^{8}$ The presence of hyalinized stroma was a common feature of CCC and the present case. Due to the negative mucicarmine staining and presence of ameloblastomatous islands in the current case, the diagnosis of CCC was eliminated.

Epithelial myoepithelial carcinoma is rare biphasic tumor of salivary gland. It arises most frequently in the parotid gland with female predilection. Histologically, it is composed of two cell types-an outer layer of clear cells and an inner layer of duct lining cuboidal cells. The cells are polygonal with centrally placed nuclei, clear cytoplasm, and the cause for clearing is attributed to the presence of glycogen. These clear cells are negative for PAS and are diastase-resistant. ${ }^{8,12}$ The present case showed significant positivity for PAS with diastase sensitivity (light pink), thereby excluding the diagnosis of EMC.

Clear cell variant of calcifying epithelial odontogenic tumor is an uncommon benign odontogenic tumor with aggressive clinical course and increased recurrence rate (22\%). ${ }^{14,15}$ It is one of the important differential diagnosis in which the cause for cytoplasmic clearing in tumor cells is a degenerative process or cytodifferentiation. It presents with a well-circumscribed pseudoglandular pattern with abundant clear cells and amyloid deposits. The clear cells are pale staining with dark nuclei, and these cells also are positive for PAS staining with diastase sensitivity. Amyloid deposits present in the stroma are positive for Congo red staining. ${ }^{8,12}$ The present case showed no calcifications and absence of polyhedral epithelial cells with centrally placed hyperchromatic nuclei that helped in ruling out the diagnosis of CCCEOT even without carrying out the Congo red staining.

Clear cell odontogenic ghost cell tumor is a rare malignant odontogenic tumor that occurs more frequently in maxilla with male preponderance. It is characterized by odontogenic islands with ameloblastic-like cells and ghost cells with varying amounts of dysplastic dentin. Clear cell change in CCOGCT is very rare, and the cause for cytoplasmic clearing is hypothesized to be aberrant degeneration of odontogenic epithelial cells. ${ }^{14-16}$ The cells usually display strong positivity for Van Gieson staining with mild positivity for PAS. However, the present case displayed no ghost cells and was negative for Van Gieson, thus excluding the diagnosis of CCOGCT (Fig. 4B).

Clear cell odontogenic carcinoma is a rare malignant odontogenic tumor with debatable histogenesis. Some authors suggest that clear cell lesions in the jaw develop from cell rests of Malassez or dental lamina. It occurs commonly in females in the sixth decade of life and frequently affects the mandible as compared to the maxilla. Clinically, it presents as an asymptomatic slow-growing swelling. ${ }^{1,2}$ The clear cells in CCOCa mimic the presecretory phase of ameloblasts. Histopathologically, it has three patterns, namely monophasic, biphasic, and ameloblastomatous. The classic cellular feature is the presence of clear cells that show central or eccentrically placed nuclei with abundant cytoplasm comprising of glycogen predominantly. ${ }^{8}$ The present case showed biphasic pattern of clear cells with dark peripherally placed nuclei and polyhedral squamous cells. The clear cells were negative for mucicarmine and Van Gieson and positive for PAS with diastase sensitivity (Figs $4 C$ and D). With these special stains, a final diagnosis of CCOCa was confirmed.

According to literature search and best of our knowledge, 125 cases of CCOCa have been reported till date. ${ }^{8}$ There have been minimal cases reported till date in males with such extensive involvement and histopathologically displaying squamous metaplasia and keratin pearls making this a unique case. ${ }^{5,6,12}$ Literature review reveals a recurrence rate of $55 \%$ and metastasis of $31 \%$ for CCOCa, thereby demanding an aggressive treatment approach with long-term follow-up. The treatment of choice for CCOCa is surgical resection with wide margins, and for cases showing perivascular and perineural invasion, adjuvant radiotherapy has to be provided. Conservative surgery generally leads to a high rate of recurrence. ${ }^{8}$ Therefore, surgical resection of the tumor with wide margins was proposed for the present case.

\section{Conclusion}

Differentiating COCCa from the other clear cell tumors of the head and neck region is difficult due to overlapping histopathology. It is necessary to distinguish CCOCa from other clear cell lesions as it has serious consequences on the biological behavior of the lesion due to its aggressiveness. In the present case, diagnostic dilemma was resolved with the use of suitable special stains that played a major role in the diagnosis of CCOCa. A close and long-term follow up is necessary to avoid recurrences.

\section{References}

1. Reichart PA, Philipsen HP. Odontogenic tumors and allied lesions. Quintessence Pub.; 2004 Jan.

2. Nazir H, Salroo IN, Mahadesh J, et al. Clear cell entities of the head and neck: A histopathological review. IOSR J Dent Med Sci 2015;14(6): 125-135.

3. Siraj F, Kaur M, Agrawal U. Clear cell odontogenic carcinoma of maxilla: A diagnostic challenge. Clin Cancer Invest J 2016 May 1;5(3):256-258.

4. Premalatha BR, Rao RS, Patil S, et al. Clear cell tumors of the head and neck: an overview. World J Dent 2012 Oct;3(4):344-349. DOI: 10.5005/ jp-journals-10015-1187.

5. Elbeshir El, Harris M, Barrett AW. Clear cell odontogenic carcinoma of the maxilla: clinical, histological and immunohistochemical features of a case. Oral Oncol 2004 Sep 1;40(8-9):91-94. DOI: 10.1016/ j.ooe.2004.05.004.

6. Jayapalan CS, George A, Noufal A, et al. Clear Cell Odontogenic Carcinoma (CCOC): Mini-Review of Literature and Case Report of Mandibular Radiolucency in 17-year Girl. Diagn Pathol Open 2016;1(2):120. 
7. Kwon IJ, Kim SM, Amponsah EK, et al. Mandibular clear cell odontogenic carcinoma. World J Surg Oncol 2015 Dec;13(1):284. DOI: 10.1186/s12957-015-0693-4.

8. Datar UV, Kamat MS, Kanitkar SS, et al. Clear cell odontogenic carcinoma: A rare case report with emphasis on differential diagnosis. J Cancer Res Ther 2017 Apr 1;13(2):374-377. DOI: 10.4103/09731482.187381.

9. Swain N, Dhariwal R, Ray JG. Clear cell odontogenic carcinoma of maxilla: A case report and mini review. J Oral Maxillofac Pathol 2013;17(1):89-94. DOI: 10.4103/0973-029X.110681.

10. Krishnamurthy A, Ramshankar V, Majhi U. Clear cell odontogenic carcinoma of the mandible and temporomandibuar joint with cervical lymph nodal metastasis. Natl J Maxillofac Surg 2014 Jul;5(2):221-223. DOI: 10.4103/0975-5950.154842.

11. Kumamoto H, Yamazaki S, Sato A, et al. Clear cell odontogenic tumor in the mandible: report of a case with duct-like appearances and dentinoid induction. J Oral Pathol Med 2000 Jan;29(1):43-47. DOI: 10.1034/j.1600-0714.2000.290108.x.
12. Avninder S, Rakheja D, Bhatnagar A. Clear cell odontogenic carcinoma: a diagnostic and therapeutic dilemma. World J Surg Oncol 2006 Dec;4(1):91. DOI: 10.1186/1477-7819-4-91.

13. Varma S, Shameena PM, Sudha S, et al. Clear cell variant of intraosseous mucoepidermoid carcinoma: report of a rare entity. J Oral Maxillofac Pathol 2012 Jan;16(1):141-144. DOI: 10.4103/0973029X.92994.

14. Turatti E, Brasil J.Clear cell variant of calcifying epithelial odontogenic tumor: Case report with immunohistochemical findings. J Clin Exp Dent 2015 Feb;7(1):e163-e166. DOI: 10.4317/jced.51995.

15. Chatterjee RP, Gayen S, Kundu S, et al. A unique case of clear cell variant of calcifying epithelial odontogenic tumor involving the maxilla. Dent Res J 2017 Jul;14(4):293-296. DOI: 10.4103/17353327.211623.

16. Yoon JH, Ahn SG, Kim SG, et al. Odontogenic ghost cell tumour with clear cell components: clear cell odontogenic ghost cell tumour? J Oral Pathol Med 2004 Jul;33(6):376-379. DOI: 10.1111/j.16000714.2004.00200.x. 